\title{
PANCREATITIS
}

\section{Neural alterations in surgical stage chronic pancreatitis are independent of the underlying aetiology}

\author{
H Friess, S Shrikhande, M Shrikhande, M Martignoni, C Kulli, A Zimmermann, \\ A Kappeler, H Ramesh, M Büchler
}

Gut 2002;50:682-686

\begin{abstract}
Background and aims: Among various causes, nerve alterations and neuroimmune interactions have been suggested to participate in the generation of pain in chronic pancreatitis (CP). In this study, we compared neural changes and the pattern of perineural inflammatory cell infiltrates in three different aetiological forms of CP (alcoholic, idiopathic, and tropical) and evaluated whether differences exist between these groups.

Patients and methods: A total of 35 patients with CP (12 tropical, 12 idiopathic, and 11 alcoholic) were included. Ten normal pancreatic tissues obtained from healthy organ donors served as controls. In all samples, the number of nerves, area of neural tissue, nerve size, and percentage of neural tissue and perineural inflammatory cell infiltrates were analysed histologically. Results: The median number of nerves per $10 \mathrm{~mm}^{2}$ tissue area was $2.3,4.3,4.4$, and 2.6 in the normal pancreas, alcoholic $C P$, idiopathic $C P$, and tropical $C P$, respectively. Median area of neural tissue per $10 \mathrm{~mm}^{2}$ was 2550, 21803,18595 , and 24666 m $^{2}$ in the normal pancreas, alcoholic CP, idiopathic $C P$, and tropical $C P$, respectively. Median nerve diameter was $36.85 \mu \mathrm{m}$ in the normal pancreas, $80.6 \mu \mathrm{m}$ in alcoholic $\mathrm{CP}, 68.95 \mu \mathrm{m}$ in idiopathic $\mathrm{CP}$, and $93.05 \mu \mathrm{m}$ in tropical $\mathrm{CP}$. In comparison with normal controls, all of these parameters were significantly increased except the number of nerves in tropical CP. For all parameters there were no significant differences between alcoholic, idiopathic, and tropical CP. When the degree of perineural inflammation was evaluated, no differences were observed among the three CP groups.

Conclusions: Independent of the underlying aetiology, CP is associated with an increase in neural tissue, and neural alterations occur in a similar fashion irrespective of the type of initiating event.
\end{abstract}

See end of article for authors' affiliations

Correspondence to: Dr H Friess, Department of General Surgery, University of Heidelberg, Im

Nevenheimer Feld 110

D-69120 Heidelburg

Germany; Helmut_Friess@ med.uni-heidelberg.de

Accepted for publication 6 August 2001

C hronic pancreatitis (CP) is an inflammatory disease primarily of the exocrine pancreas which leads to persistent progressive morphological alterations and physiological dysfunction. ${ }^{1-3}$ In the past few years several aetiological factors of $\mathrm{CP}$ have been recognised, contributing to a better understanding of the disease. We have learnt that mutations in the cationic trypsinogen gene are present in patients with hereditary $\mathrm{CP}$, and it has been reported that approximately $30 \%$ of patients with so-called idiopathic CP have mutations in the cystic fibrosis transmembrane conductance regulator gene. ${ }^{4-7}$ Although the morphological changes in $\mathrm{CP}$ have been recognised and described for many decades, the pathophysiology of CP is still poorly understood and it is not known whether the pathophysiological events in different CP aetiologies are similar or diverse.

In Western industrialised countries, the dominant aetiological factor of $\mathrm{CP}$ is alcohol abuse, accounting for approximately $80 \%$ of patients. ${ }^{8}$ In a small percentage of patients with $\mathrm{CP}$, alcohol abuse can be clearly ruled out as an aetiological factor, and if other known aetiological factors are absent, these patients are then classified as having idiopathic $\mathrm{CP}^{8-10}$ In contrast with Western countries, in Southern India an alcohol independent form of $\mathrm{CP}$-so-called tropical pancreatitis-is common. ${ }^{8}$ Here there is a chronic calcifying form of $\mathrm{CP}$ in a younger age group in a region of high incidence without any history of alcohol consumption. The aetiology of tropical CP is largely unknown, and nutritional and environmental factors have been propagated since the 1970s and 1980s as major causative factors in the aetiopathogenesis of this form of $\mathrm{CP}^{11}{ }^{12}$

In alcoholic $\mathrm{CP}$, changes involving neural proliferation with the resultant increase in number of nerves and nerve diameter, damage to the perineurium, increase in sensory neurotransmitters, and a neuroimmune interaction of inflammatory cells with altered nerves were reported recently. ${ }^{13-16}$ Furthermore, these changes were suggested to be involved in pain in alcoholic CP. ${ }^{16}$ However, idiopathic ${ }^{17}$ and tropical ${ }^{18} \mathrm{CP}$ are also associated with pain. Therefore, if nerves are involved in the pathogenesis of pain in $\mathrm{CP}$, similar neural alterations should be present in $\mathrm{CP}$, independent of the underlying aetiology. Similar neural changes would provide further evidence with regard to the significance of neural alterations in the pain pathogenesis of CP. Therefore, in the present study, neural changes were analysed and compared in three different aetiological forms of CP: alcoholic, idiopathic, and tropical CP. As inflammatory cell infiltrates are regularly found in alcoholic CP and a close interaction between inflammatory cells, pancreatic nerves, and the presence of pain was recently reported, ${ }^{16}$ perineural inflammatory cell infiltrates were also evaluated

\section{PATIENTS AND METHODS}

Patients

Pancreatic tissue samples of 35 patients $(25$ males and 10 females; mean age 37 (11.8) years) with CP and pancreatic resection were included in the study. No duct samples were included and only acinar tissue was used in this study. Aetiology was alcohol in 11 patients (nine males, two females; mean age 43.8 (8.1) years) and idiopathic in 12 patients (six males, six females; mean age 33.5 (15.1) years). While all had

Abbreviations: $\mathrm{CP}$, chronic pancreatitis; PGP 9.5, protein gene product 9.5; TBS, Tris buffered saline. 
Table 1 Comparison of nerve related parameters in the different aetiological groups of chronic pancreatitis (CP)

\begin{tabular}{|c|c|c|c|c|}
\hline Parameter & Normal pancreas & Alcoholic CP & Idiopathic CP & Tropical CP \\
\hline $\begin{array}{l}\text { Median tissue area analysed } \\
\left(\mathrm{mm}^{2}\right)\end{array}$ & $80.9(61-125)$ & 97.9 (57.9-149.7) & $62(42.3-85.9)$ & 120.5 (77.-174.7) \\
\hline $\begin{array}{l}\text { Median No of nerves per } \\
10 \mathrm{~mm}^{2} \text { tissue area }\end{array}$ & $2.3(1.2-2.8)$ & $4.3(2.4-5.6)$ & $4.4(3.9-6.6)$ & $2.6(2.2-3.9)$ \\
\hline $\begin{array}{l}\text { Median innervation area per } \\
\text { nerve }\left(\mathrm{mm}^{2}\right)\end{array}$ & $4.3(3.5-8.0)$ & $2.3(1.8-4.2)$ & $2.3(1.5-2.6)$ & $3.9(2.5-4.5)$ \\
\hline $\begin{array}{l}\text { Median area of nerves per } 10 \\
\mathrm{~mm}^{2} \text { tissue area }\left(\mu \mathrm{m}^{2}\right)\end{array}$ & $2550(1474-3506)$ & 21803 (10382-39554) & 18595 (1 1808-43263) & 24666 (14438-46589) \\
\hline Median nerve area $\left(\mu \mathrm{m}^{2}\right)$ & $1068.6(923.5-1404.07)$ & $5110.8(3126.1-10832.1)$ & 3743.9 (2680-9951.1) & $6797.9(5258.6-16910.0)$ \\
\hline Median nerve diameter $(\mu \mathrm{m})$ & $36.8(34.2-42.2)$ & $80.6(63.1-117.4)$ & $68.9(58.3-112.5)$ & 93.0 (81.7-146.3) \\
\hline $\begin{array}{l}\text { Median percentage of neural } \\
\text { tissue }(\%)\end{array}$ & $0.025(0.014-0.035)$ & $0.21(0.10-0.39)$ & $0.18(0.11-0.43)$ & $0.24(0.14-0.46)$ \\
\hline
\end{tabular}

an inflammatory mass in the pancreatic head, there was some obstructive element with ductal dilatation in all patients except one. All patients with alcoholic and idiopathic CP were operated on at the Inselspital, University of Bern. Pancreatic tissue from 12 patients with tropical CP (10 males, two females; mean age 33.8 (9.2) years) who were operated on in India were also included. While eight tropical CP patients had a dilated duct disease, four had an inflammatory mass in the head of the pancreas. The degree of ductal dilatation was in the range $7-10 \mathrm{~mm}$. The indication for surgery, both in Bern and in India, was uncontrolled pain and patients had either an inflammatory mass in the head of the pancreas leading to obstructive complications on pancreatic neighbouring organs, a dilated main pancreatic duct, or a combination of both. Overall, no major differences were observed between European and Indian subjects. For patients with idiopathic CP, alcohol abuse and other known causes of CP were ruled out. The same was true for Indian patients with tropical CP. Additionally, 10 normal human pancreatic tissue samples, obtained through an organ donor programme, served as controls. There were five male and five female organ donors (mean age 44.4 (17.1) years). The studies were approved by the human subjects committee of the University of Bern.

\section{Collection of clinical parameters}

In all patients operated on at the Inselspital in Bern (alcoholic and idiopathic $\mathrm{CP}$ ), the clinical patient parameters were collected prospectively in a computer assisted database and pain history was evaluated in the year before operation. Pain intensity was classified into four categories: absent (score 0 ), mild (score 1), moderate (score 2 ), and severe (score 3$){ }^{16}$. The frequency of pain was defined as daily (score 3), weekly (score 2 ), and monthly (score 1). ${ }^{16}$ In addition, duration of pain was recorded and classified into three categories: short (score 1; 0-90 days), medium ( score 2; 91-365 days), and long (score 3; more than 365 days).

For patients operated on for tropical CP in India, the intensity, frequency, and duration of pain were recorded in an identical fashion. A global pain score was calculated for each patient by adding the scores for intensity, frequency, and duration.

\section{Immunohistochemistry of PGP 9.5-identification of neural tissue}

PGP 9.5 (protein gene product 9.5) immunostaining was used to specifically stain neural tissue, as described previously. ${ }^{15}{ }^{16}$ Consecutive 3-5 $\mu \mathrm{m}$ paraffin embedded tissue sections were subjected to immunostaining using the peroxidase technique. ${ }^{19}{ }^{20}$ After deparaffinisation and rehydration, tissue sections were submerged for 15 minutes in Tris buffered saline (TBS) solution ( $10 \mathrm{mM}$ Tris $\mathrm{HCl}, 0.85 \% \mathrm{NaCl}, \mathrm{pH} 7.4$ ) containing $0.1 \%$ (vol/vol) Triton X-100 and then washed for five minutes in TBS buffer. Endogenous peroxidase activity was blocked by incubating the slides in methanol and in methanol/2\% hydrogen peroxide, followed by washings in methanol and twice in TBS containing $0.1 \%$ bovine serum albumin. Sections were then incubated for 30 minutes at $37^{\circ} \mathrm{C}$ with $10 \%$ normal goat serum prior to overnight incubation. Incubation was carried out at $4^{\circ} \mathrm{C}$, with the primary antibody diluted in $10 \%$ normal goat serum as follows: polyclonal rabbit anti-PGP 9.5 (Ultraclone Ltd, U.K) (1:1000 dilution) raised against human PGP 9.5 protein purified from pathogen free human brain. Bound antibody was detected with a biotinylated goat antirabbit IgG and a streptavidin-peroxidase complex (Kirkegaard and Perry Laboratories, Inc., Gaithersburg, Maryland, USA), followed by incubation with diaminobenzidine tetrahydrochloride $(0.05 \%)$ as substrate and counterstaining with Mayer's haematoxylin. To ensure the specificity of the primary antibodies, consecutive tissue sections were incubated either in the absence of the primary antibody or with a non-immunised rabbit IgG antibody. In these sections no immunostaining was detected.

\section{Quantitative analysis of neural tissue}

Analysis of pancreatic nerves was carried out by two independent observers with the help of digital image analysis and the Image Pro-plus software (Media Cybernetics, Silver Spring, Maryland, USA). A charge coupled device video camera module and PROSCAN electronics 5000 (Hama, Germany) connected to the Image Pro-plus version 3.1 software (Media Cybernetics) were used to measure the entire area of each tissue section, number of nerves, and nerve area. From the recorded measurements, average area of nerves per $10 \mathrm{~mm}^{2}$ tissue area and the percentage of tissue occupied by nerves were calculated. Also, innervation area per nerve and number of nerves per $10 \mathrm{~mm}^{2}$ of tissue area were calculated.

\section{Evaluation of inflammatory cell infiltrates}

The tissue sections were also evaluated for perineural inflammatory cell infiltrates by two independent observers blinded to patient status, followed by joint resolution of any differences. Furthermore, infiltrate density was graded as mild (score 1), moderate (score 2), or severe (score 3). For the purposes of comparative evaluation between the different groups, the area of inflammatory foci was measured with a video image system. To summarise the degree of perineural inflammation in CP tissues, a perineural inflammation number score and a perineural area score were calculated, taking into account the total number of perineural inflammatory foci, density of the cell infiltrates, area of foci, and area of the tissue sections evaluated. Hence the perineural inflammation number score was defined as:

(No of perineural foci with density score $1 \times 1)+($ No of perineural foci with density score $2 \times 2$ ) + (No of perineural foci with density score $3 \times 3) \times 1000 /$ Area of tissue evaluated $\left(\mathrm{mm}^{2}\right)$.

The perineural inflammation area score was defined as: (Mean area of perineural foci with density score $1 \times 1)+$ 


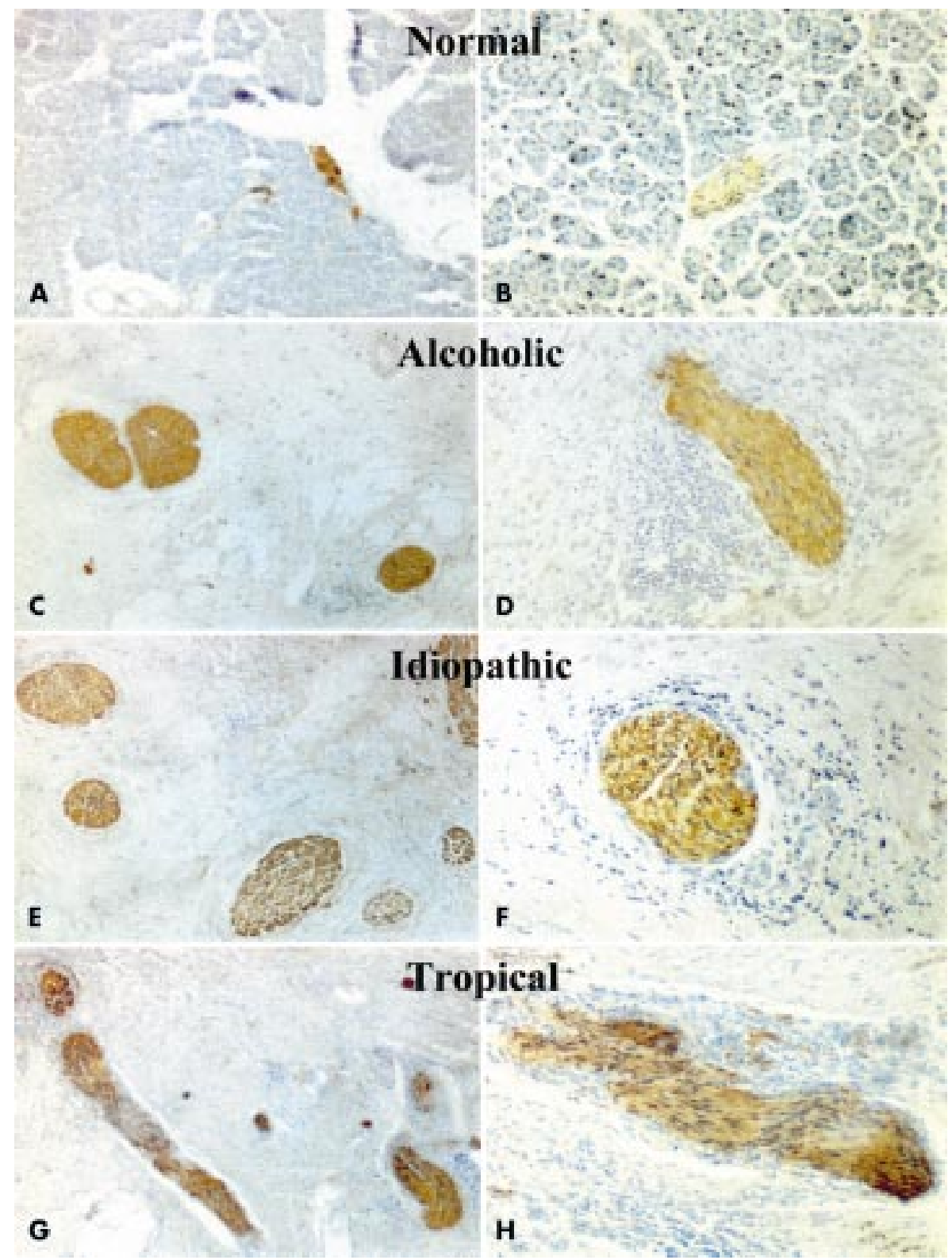

Figure 1 Protein gene product 9.5 (PGP 9.5) immunostaining in the normal pancreas $(A, B)$, alcoholic chronic pancreatitis $(C P)(C, D)$, idiopathic $C P(E, F)$, and tropical $C P(G, H)$. The left panel shows an original magnification of $\times 125$ and the right side panel an original magnification of $\times 250$. In alcoholic, idiopathic, and tropical $C P$, a comparable increase in neural tissue was present. Furthermore, perineural inflammatory cell infiltrates were present in a similar number and size in all three aetiological forms of CP.

(Mean area of perineural foci with density score $2 \times 2)+$ (Mean area of perineural foci with density score $3 \times 3) \times 10^{6} \%$ Area of tissue evaluated $\left(\mathrm{mm}^{2}\right)$

where mean area of perineural foci was measured in $\mathrm{mm}^{2}$.

Also, the percentage involvement of the perineural infiltrate was evaluated and summarised for each patient group.

\section{Evaluation of pancreatic fibrosis}

The degree of pancreatic fibrosis was evaluated on haematoxylin-eosin stained tissue sections by the use of digital image analysis and the Image Pro-plus software (Media Cybernetics). For further analysis, fibrosis was grouped as mild $(<25 \%)$, moderate $(\geqslant 25-50 \%)$, severe $(\geqslant 50-75 \%)$, and marked $(\geqslant 75 \%)$.

\section{Statistical analysis}

Statistical analysis was performed with the SPSS 9/0 statistical program. Data are given as mean (SD) or median (upper/ lower quartiles). For statistical analysis, the Kruskal-Wallis test, Mann-Whitney U test, and Spearman correlation analysis were used. A p value $<0.05$ was taken as significant.

\section{RESULTS}

The pain history of patients with alcoholic, idiopathic, and tropical CP was similar $(p=0.13)$.

In alcoholic CP, four patients had severe pain, four had moderate pain, and three had mild pain. While two patients had daily pain, five had weekly pain, and four had monthly pain. The median (25th-75th percentiles) global pain score in alcoholic CP was 6 (6-7)/patient.

In idiopathic CP, two patients had severe pain, six had moderate pain, and four had mild pain. While four patients had daily pain, five had weekly pain, and three had monthly pain. The median (25th-75th percentiles) global pain score in idiopathic CP was 6 (6-7)/patient. 
Table 2 Perineural inflammation number score and perineural inflammation area score in different aetiological groups of chronic pancreatitis (CP)

\begin{tabular}{|c|c|c|c|c|c|}
\hline Aetiology & Inflammation No score & Inflammation area score & Intensity of pain & Frequency of pain & Global pain score \\
\hline Alcoholic CP & $70(44-106)$ & $512(300-831)$ & $2(1-3)$ & $2(1-2)$ & $6(6-7)$ \\
\hline Idiopathic CP & $80(0-113)$ & $300(0-1340)$ & $2(1-2)$ & $2(1.25-3)$ & $6(6-7)$ \\
\hline Tropical CP & 61 (19-153) & $613(78-1366)$ & $3(3-3)$ & $2(1-2.75)$ & $7(7-8)$ \\
\hline
\end{tabular}

In tropical $\mathrm{CP}, 11$ patients had severe pain and one had moderate pain. While three patients had daily pain, four had weekly pain, and five had monthly pain. The median (25th-75th percentiles) global pain score in tropical CP was 7 (7-8)/patient.

\section{Evaluation of pancreatic nerves}

The median (upper/lower quartiles) analysed tissue area, median number of nerves per $10 \mathrm{~mm}^{2}$ of tissue area, median innervation area per nerve, median area of nerves per $10 \mathrm{~mm}^{2}$ area of tissue, median nerve area, median nerve diameter, and median percentage of neural tissue in normal pancreas, alcoholic, idiopathic, and tropical CP are given in table 1.

\section{Comparison of neural changes between normal and alcoholic, idiopathic, and tropical CP (fig 1)}

Comparison of alcoholic CP with normal controls revealed that while the median number of nerves $/ 10 \mathrm{~mm}^{2}$ of tissue area was marginally higher $(p=0.051)$ than in normal controls, the median area of nerves $/ 10 \mathrm{~mm}^{2}$ of tissue area $(p<0.001)$, median nerve area $(\mathrm{p}<0.001)$, median nerve diameter $(\mathrm{p}<0.001)$, and percentage of tissue area occupied by nerves $(\mathrm{p}<0.001)$ were significantly higher in alcoholic CP than in normal controls.

Comparison of idiopathic CP with normal controls revealed that the median number of nerves $/ 10 \mathrm{~mm}^{2}$ tissue $(p=0.003)$, median area of nerves $/ 10 \mathrm{~mm}^{2}$ of tissue area $(\mathrm{p}<0.001)$, median nerve area $(\mathrm{p}<0.001)$, median nerve diameter $(\mathrm{p}<0.001)$, and percentage of tissue occupied by nerves $(p<0.001)$ were significantly higher in idiopathic CP than in normal controls.

Comparison of tropical CP with normal controls revealed that while the median number of nerves/10 $\mathrm{mm}^{2}$ tissue $(p=0.283)$ was nearly the same as in normal controls, median area of nerves $/ 10 \mathrm{~mm}^{2}$ of tissue area $(\mathrm{p}<0.001)$, median nerve area $(p<0.001)$, median nerve diameter $(p<0.001)$, and percentage of tissue occupied by nerves $(\mathrm{p}<0.001)$ were significantly higher in tropical CP than in normal controls.

\section{Comparison of neural findings in different aetiologies of CP}

Median innervation area per nerve was significantly higher in tropical CP compared with idiopathic CP $(p=0.02)$. Therefore, the number of nerves $/ 10 \mathrm{~mm}^{2}$ of tissue area was significantly higher in idiopathic CP in comparison with tropical CP $(\mathrm{p}=0.02)$.

For all other parameters-including median number of nerves $/ 10 \mathrm{~mm}^{2}$ tissue area, median area of nerves $/ 10 \mathrm{~mm}^{2}$ of tissue area, median nerve area, median nerve diameter, and percentage of tissue occupied by nerves-no significant differences were observed between alcoholic, idiopathic, and tropical CP.

\section{Evaluation of perineural inflammatory cell infiltrates in different aetiologies of CP}

All tissue sections of the normal pancreas had no perineural inflammatory cell infiltrates. In contrast, in alcoholic, idiopathic, and tropical CP, various degrees of perineural cell infiltrates were present. However, comparison of the perineural inflammation number score revealed no differences between alcoholic and idiopathic CP $(\mathrm{p}=0.26)$, between alcoholic and tropical CP $(\mathrm{p}=0.928)$, or between idiopathic and tropical CP $(p=0.29)$ (table 2$)$. When the perineural inflammation area scores were compared in the different aetiological groups, again no differences were present between alcoholic and idiopathic CP $(p=0.08)$, between alcoholic and tropical CP $(p=0.88)$, or between idiopathic and tropical CP $(p=0.18)$ (table 2).

The percentage involvement of perineural foci was also evaluated in the different aetiological groups and no significant differences between the three groups were found $(\mathrm{p}=0.37)$.

\section{Comparison of pancreatic fibrosis in different aetiologies of $\mathrm{CP}$}

There was no relationship $(p>0.05)$ between the degree of pancreatic fibrosis and pain in any aetiological CP group.

\section{DISCUSSION}

A common dominant feature of $\mathrm{CP}$, independent of the underlying aetiology, is abdominal pain. In the past few years, various hypotheses have been implicated in the pathogenesis of pain in CP; however, none has firmly stood the test of time. Increased intraductal pressure as a result of strictures and/or calculi is believed to be the most common cause of pain for patients with dilated main pancreatic duct disease, and relief from pain by drainage of a dilated main pancreatic duct is often seen..$^{21}$ The other suggested causes of pain are duodenal and common bile duct stenosis due to ongoing pancreatic inflammation and subsequent scarring. ${ }^{23}$ Additionally, pancreatic fibrosis, interstitial hypertension, and pancreatic ischaemia have all been implicated either as a sole or additional factors involved in the pathogenesis of pain. ${ }^{24} 25$

The most recent concept of pain pathogenesis in alcoholic $\mathrm{CP}$ postulates that alterations in pancreatic nerves and neuroimmune interactions are crucial factors in the pain syndrome. ${ }^{1626}$ Pancreatic nerves are preferentially retained while the parenchyma degenerates and is replaced by fibrotic tissue. Increased diameter of pancreatic nerves, damage to the perineurium, ${ }^{14}$ and increased presence of neurotransmitters such as substance P and CGRP in enlarged pancreatic nerves in CP suggests that pancreatic nerves might be responsible for or are at least one of the main players in the long lasting pain syndrome in these patients. ${ }^{15}$ However, if nerves are involved in the pathogenesis of pain in alcoholic CP, similar neural alterations should also be present in other types of CP associated with pain, independent of the underlying aetiology. Inasmuch as pain is a dominant feature of alcoholic, idiopathic, and tropical CP, the presence of similar neurological alterations and their relationship to pain would support the significance of neural alterations in the pain pathogenesis of CP. In our study, patients of different aetiological groups had a comparable pain syndrome with regard to pain intensity, frequency, and duration, and exhibited similar neural alterations with an increase in neuronal tissue, supporting the hypothesis that changes in innervation are of importance in the pain syndrome of CP. The only statistically significant difference was noted between non-tropical CP and tropical CP in terms of median number of nerves per tissue area and consequently median innervation area per nerve. In tropical CP, the 
median number of nerves per tissue area was significantly less compared with that in idiopathic CP and was also less compared with alcoholic CP although this was not statistically significant. As a natural consequence, median innervation area per nerve was more in tropical CP than in other forms of CP. A possible explanation could be that the individual nerve diameter in tropical CP, although not statistically significant, showed a definite trend towards being larger than in other forms of CP. Thus nerves appear to enlarge rather than proliferate in tropical CP. The final outcome being the amount of neuronal tissue, it is possible that either nerve enlargement or proliferation or likely a combination of both are responsible for the pain syndrome of CP.

In 1985, attention was focused on immune cell infiltrates which are frequently located close to pancreatic nerves in alcoholic CP. ${ }^{13}$ This histopathological study suggested that neuroimmune mechanisms are probably involved in the pain of CP. The study stimulated further research on the combined role of nerves and the immune system in the pathogenesis of this disorder. More recently, it was demonstrated that interaction of immune cells with pancreatic nerves and a marked increase in neuronal plasticity are likely additional pathogenic factors for the generation of pain in $\mathrm{CP}$ whereas the degree of pancreatic fibrosis has no major impact on the pain syndrome in these patients. ${ }^{16}$ Therefore, as inflammatory cell infiltrates are regularly found in alcoholic $\mathrm{CP}$ and a close interaction between inflammatory cells, pancreatic nerves, and the presence of pain has been observed, we also evaluated and compared the pattern of perineural inflammatory cell infiltrates in other aetiological groups of CP. Again we found a similar pattern of perineural inflammatory cell infiltrates, irrespective of the aetiological initiation of the disease. These findings, considered together, indicate that comparable neural alterations occur in painful CP independent of the underlying aetiology and possibly explain the similar course of pain observed in alcoholic and non-alcoholic CP. ${ }^{17}{ }^{18}$

A limitation of our study is that all pancreatic tissues were obtained from CP patients with "surgical stage $\mathrm{CP}^{\text {" - }}$-the only stage when surgical intervention is considered necessary. Due to the lack of non-surgical stage human CP tissue and the unavailability of relevant experimental models of CP that mimic the aetiology and other features associated with human $\mathrm{CP}$, the development of neural alterations and the relationship with perineural inflammatory cell infiltrates cannot be studied in a time dependent fashion.

Nevertheless, while there are reasons to believe that different forms of CP follow different courses depending on the initiating aetiological event, our findings suggest that as the disease seems to approach a common stage where the neural alterations and the pattern of perineural inflammation are similar irrespective of the underlying aetiology, the activated pathobiological pain pathways may ultimately follow a distinctive, aetiology independent pattern in all forms of CP.

\section{Authors' affiliations}

H Friess, S Shrikhande, M Shrikhande, M Martignoni, C Kulli, M

Büchler, Department of Visceral and Transplantation Surgery, University of Bern, Inselspital, Bern, Switzerland
A Zimmermann, A Kappeler, Institute of Pathology, University of Bern, Inselspital, Bern, Switzerland

H Ramesh, PVS Memorial Hospital, Cochin, India

\section{REFERENCES}

1 Sarles H, Bernard JP, Johnson C, et al. Pathogenesis and epidemiology of chronic pancreatitis. Ann Rev Med 1989;40:453-68.

2 DiMagno EP. Conservative management of chronic pancreatitis. Dig Surg 1994;11:300-3.

3 DiMagno EP. A short, eclectic history of exocrine pancreatic insufficiency and chronic pancreatitis. Gastroenterology 1993; 104:1255-62.

4 Cohn JA, Friedman KJ, Noone PG, et al. Relation between mutations of the cystic fibrosis gene and idiopathic pancreatitis. N Engl J Med 1998:339:653-8.

5 Cohn JA, Jowell PS. Are mutations in the cystic fibrosis gene important in chronic pancreatitis? Surg Clin North Am 1999;79:723-31.

6 Choudari CP, Lehman GA, Sherman S. Pancreatitis and cystic fibrosis gene mutations. Gastroenterol Clin North Am 1999;28:543-9.

7 Taylor CJ. Chronic pancreatitis and mutations of the cystic fibrosis gene. Gut 1999;44:8-9.

8 Pitchumoni CS. Chronic pancreatitis: a historical and clinical sketch of the pancreas and pancreatitis. Gastroenterologist 1998;6:24-33.

9 Ammann R. Idiopathic juvenile chronic pancreatitis. Disch Med Wochenschr 1976;101:1789-94.

10 Ammann R, Sulser H. "Senile" chronic pancreatitis; a new nosologic entity? Studies in 38 cases. Indications of a vascular origin and relationship to the primarily painless chronic pancreatitis. Schweiz Med Wochenschr 1976;106:429-37.

11 Nwokolo C, Oli J. Pathogenesis of juvenile tropical pancreatitis syndrome. Lancet 1980;1:456-9.

12 Braganza JM, Schofield D, Snehalatha C, et al. Micronutrient antioxidant status in tropical compared with temperate-zone chronic pancreatitis. Scand J Gastroenterol 1993;28:1098-104.

13 Keith RG, Keshavjee SH, Kerenyi NR. Neuropathology of chronic pancreatitis in humans. Can J Surg 1985;28:207-11.

14 Bockman DE, Büchler M, Malfertheiner $P$, et al. Analysis of nerves in chronic pancreatitis. Gastroenterology 1988;94:1459-69.

15 Büchler $M$, Weihe $E$, Friess $H$, et al. Changes in peptidergic innervation in chronic pancreatitis. Pancreas 1992;7:183-92.

16 Di Sebastiano P, Fink T, Weihe E, et al. Immune cell infiltration and growth-associated protein 43 expression correlate with pain in chronic pancreatitis. Gastroenterology 1997;112:1648-55.

17 Lankisch PG, Seidensticker F, Lohr-Happe A, et al. The course of pain is the same in alcohol- and non-alcohol induced chronic pancreatitis. Pancreas 1995; 10:338-41.

18 Chari ST, Mohan V, Jayanthi V, et al. Comparative study of the clinical profiles of alcoholic chronic pancreatitis and tropical chronic pancreatitis in Tamil Nadu, south India. Pancreas 1992;7:52-8.

19 Di Sebastiano P, Fink T, Weihe E, et al. Changes of protein gene product 9.5 (PGP 9.5) immunoreactive nerves in inflamed appendix. Dig Dis Sci 1995:40:366-72.

20 Friess $\mathrm{H}$, Yamanaka $Y$, Büchler $M$, et al. Increased expression of acidic and basic fibroblast growth factors in chronic pancreatitis. Am J Pathol 1994; 144:117-28.

21 Warshaw AL, Popp JW Jr, Schapiro RH. Long-term patency, pancreatic function, and pain relief after lateral pancreaticojejunostomy for chronic pancreatitis. Gastroenterology 1980;79:289-93.

22 Bradley EL 3rd. Long-term results of pancreatojejunostomy in patients with chronic pancreatitis. Am J Surg 1987;153:207-13.

23 Manes G, Büchler M, Pieramico O, et al. Is increased pancreatic pressure related to pain in chronic pancreatitis. Int J Pancreatol 1994;5: 113-17.

24 Reber HA, Karanja ND, Alvarez C, et al. Pancreatic blood flow in cats with chronic pancreatitis. Gastroenterology 1992;103:652-9.

25 Karanjia ND, Widdison AL, Leung F, et al. Compartment syndrome in experimental chronic obstructive pancreatitis: effect of decompressing the main pancreatic duct. Br J Surg 1994;81:259-64.

26 Weihe E, Nohr D, Müller S, et al. The tachykinin neuroimmune connection in inflammatory pain. Ann NY Acad Sci 1991;632:283-95. 